\title{
LANGMUIR WAVE STRUCTURES REGISTERED BY FREJA: ANALYSIS AND MODELING
}

\author{
Yu. Khotyaintsev ${ }^{1}$, G. Lizunov², K. Stasiewicz ${ }^{1}$ \\ ${ }^{1}$ Swedish Institute of Space Physics, Uppsala Division, S-75591, Sweden \\ ${ }^{2}$ Space Research Center PAS, Bartycka 18A, 00716, Warsaw, Poland
}

\begin{abstract}
A large set of observations of Langmuir emissions generated by super-thermal electron beams in the topside polar ionosphere has been obtained during the recent FREJA mission. Langmuir emissions appeared as a chain of strongly modulated wave packets; in many cases the packet envelopes were modulated periodically. In present report we suggest the theory which explains these observations. We suggest that packet-like waveforms are the beatings of several Langmuir modes. One of modes (the primary mode) appears as a result of beam-plasma instability, other modes - as a result of parametric decay of primary mode to the secondary Langmuir wave and lower-hybrid wave (or as a result of decay cascade). We present the quantitative theory of three-wave interaction $\mathrm{L} \rightarrow \mathrm{L}+\mathrm{LH}$ which explain many details of FREJA observations.
\end{abstract}

\section{MODULATED LANGMUIR EMISSIONS IN SPACE PLASMAS}

The main properties of Langmuir turbulence generated by charged particle beams in space plasmas have been established during the investigation of solar radio bursts, spectra of ionospheric and magnetospheric emissions, and active space plasma experiments. Recent satellite observations with high temporal resolution revealed that Langmuir emission appear as chains of strongly modulated wave packets. The generation of modulated Langmuir emissions has been observed in auroral ionosphere (Ergun et al, 1991), solar wind (Gurnett et al., 1993, Kellog et al., 1992), recent FREJA experiments in topside ionosphere (Stasiewicz et al., 1996, Bonnell et al., 1997), the magnetosphere (Kojima et al., 1997), as well as in laboratory plasmas (Christiansen et al., 1982) and in numerical experiments in which the space plasma conditions have been simulated (Newman et al., 1994, Akimoto et al., 1996). To explain these observations several hypotheses have been suggested: (i) Langmuir wave packets are solitons (or cavitons) similar to the laboratory results described by Nezlin (1981), (ii) Langmuir wave packets are spatial structures caused by the bounce-oscillations of trapped particles and propagating through the ambient plasma with wave group velocity (Muschietti et al., 1995, Akimoto et al., 1996), and (iii) packet-like waveforms are temporal structures produced by the beating of two or several Langmuir waves with close frequencies. The primary wave in this case is generated due to the beam-plasma instability, the secondary harmonics appear as results of parametric decay cascade (Ergun et al., 1991, Forme et al., 1993, Stasiewicz et al., 1996).

The first hypothesis (i) seems not to be valid in the case of ionospheric plasma because the generation of Langmuir wave packets is also observed at very low wave amplitudes where the nonlinear effects are unlikely to play a role. The second explanation (ii) had been carefully examined by Bonnel et al. (1997) who showed that the wave-particle trapping process predicts modulation frequency $f_{\text {Mod }} / f_{p e} \sim 10^{-4}$, which is far smaller than the modulation frequencies observed in the data set.

To focus on the third issue (iii) we show two examples from Freja data of quasi-periodical modulations (see Figure 1). The frequency spectra of Langmuir emissions are composed of several harmonics whose mixing produces various beating forms. The frequency difference of neighboring harmonics $\left(f_{1}-f_{2} \sim 10 \mathrm{kHz}\right)$ is larger than the lower-hybrid frequency of about $4 \mathrm{kHz}$ which means that Langmuir waves may be parametrically connected with lower-hybrid wave. The matching conditions for three wave interactions 


$$
\begin{aligned}
\omega_{1} & =\omega_{2}+\omega_{3} \\
\vec{k}_{1} & =\vec{k}_{2}+\vec{k}_{3}
\end{aligned}
$$

as analyzed by Stasiewicz et al. (1996), and Bonnell et al. (1997) are satisfied by a continuum of lower-hybrid modes and Langmuir modes (hereafter the indexes ' 1 ' and ' 2 ' refer to the Langmuir waves, index ' 3 ' refers to the lower-hybrid wave). Since, the waveforms on Figure 1 consist of discrete harmonics, there must be a mode selection mechanism. It has been suggested by Stasiewicz et al. (1996) that the mode selection could be related to the finite size of the electron beam region which would impose the preferred $k_{\perp}$ for lower-hybrid modes.
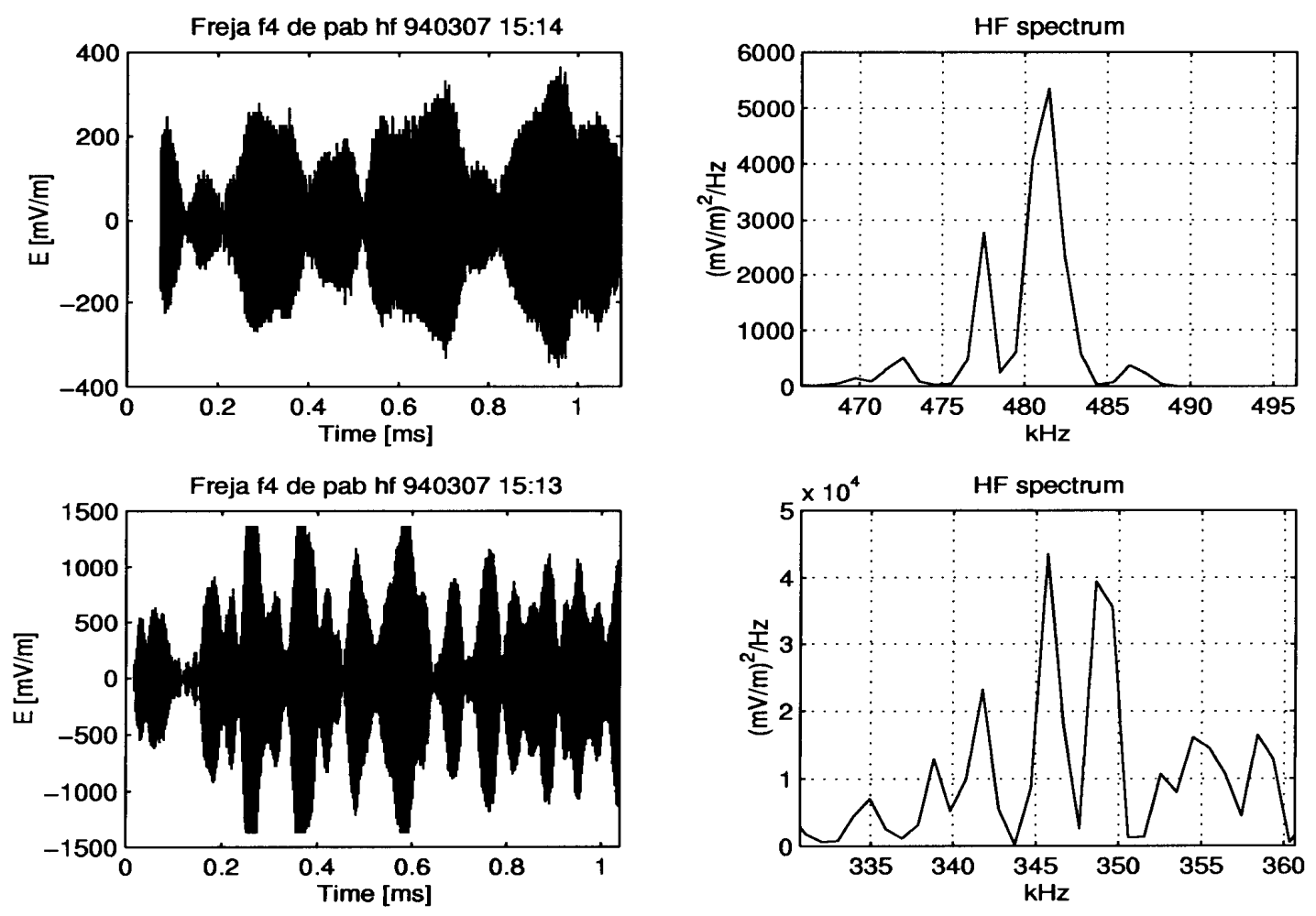

Fig.1. Waveforms (at the left) and spectral composition (at the right) of Langmuir emissions registered by FREJA

\section{BEAM-DRIVEN LANGMUIR WAVE INSTABILITY}

\subsection{Freja Observations}

The goal of the FREJA mission was the investigation of auroral plasma processes in the topside polar ionosphere. A comprehensive description of the scientific payload can be found in a special issue of Space Science Rev. 70, 405-602, (1994). We should note here that high-frequency electric field was measured in snapshots of $\sim 1$ ms duration, sampled at $8 \times 10^{6} \mathrm{~s}^{-1}$. The Langmuir frequency is typically less than the electron cyclotron frequency $\omega_{p e}<\omega_{c e}$, which introduces some peculiarities in the dispersion equation. Another important condition is that in many cases the ion temperature is higher than the electron temperature $T_{\mathrm{e}}<T_{\mathrm{i}}$ which leads to the strong damping of ion acoustic waves.

The bursts of Langmuir emissions had been observed simultaneously with the appearance of superthermal electron beams and therefore are associated with the beam-plasma instability. The registered distribution functions of superthermal electrons are wide in velocity space $\Delta V_{b} \sim V_{b}$, and the reduced "one-dimensional" distribution functions are close to the plateau or weak "bump in tail" type of distribution. However, the distribution function of superthermal electrons is registered during approximately $65 \mathrm{~ms}$ which is too long to see any evolution of distribution function caused by the beam-driven instability. In Table 1 we show the values of plasma and beam parameters observed on FREJA. At the bottom are the values of parameters used in this article. 
Table 1. Plasma Parameters of Topside Polar Ionosphere

\begin{tabular}{|c|c|c|c|c|c|c|c|}
\hline$n_{\mathrm{p},}, \mathrm{cm}^{-3}$ & $\omega_{\mathrm{p}}, \mathrm{s}^{-1}$ & $\omega_{\mathrm{c}}, \mathrm{s}^{-1}$ & $V_{\mathrm{Te}}, \mathrm{cm} / \mathrm{s}$ & $n_{\mathrm{b}}, \mathrm{cm}^{-3}$ & $\varepsilon_{\mathrm{b}}, \mathrm{eV}$ & $V_{b}, \mathrm{~cm} / \mathrm{s}$ & $\Delta V_{b}, \mathrm{~cm} / \mathrm{s}$ \\
\hline $400-3600$ & $(1-4) \times 10^{6}$ & $5 \times 10^{6}$ & $4 \times 10^{7}$ & $0.1-1$ & $10-1500$ & - & - \\
\hline 1200 & $2 \times 10^{6}$ & $5 \times 10^{6}$ & $4 \times 10^{7}$ & 1 & $10-100$ & $3 \times 10^{8}$ & $2 \times 10^{8}$ \\
\hline
\end{tabular}

\subsection{Generation of Primary Langmuir Wave}

The theoretical analysis reveals certain disagreement between calculated and observed spectra of Langmuir waves. Let's examine this problem.

According to Freja observations electron beams are weak $n_{b}<<n_{p}$ and have spread velocity spectrum $\Delta V_{b} \sim$ $V_{b}$. In this case the instability growth rate is

$$
\gamma \sim\left(\frac{V_{b}}{\Delta V_{b}}\right)^{2} \frac{n_{b}}{n_{p}} \omega
$$

where $\omega=\omega(k)$ is the frequency of Langmuir wave. The dispersion relation of Langmuir wave is

$$
\omega=\omega_{p}\left[1+3\left(k \lambda_{D}\right)^{2}-A \sin ^{2} \theta\right]^{1 / 2}
$$

where $A=1 /\left(1-\omega_{p}^{2} / \omega_{c}^{2}\right), \lambda_{\mathrm{D}}$ is Debye radii and $\theta$ is the direction of wave propagation in respect to the magnetic field. Waves generated at larger angles from the magnetic field depart from the Langmuir frequency, which contradicts to the fact that the emission spectrum consists of several narrow lines (Figure 1). An outstanding question is what determines the observed narrowness of the Langmuir wave spectrum. A similar question appeared in the article of Newman et al. (1994). The authors explained the spectrum narrowing by the Doppler damping of oblique Langmuir waves. Newman et al. (1994) have done the calculations for very specific set of ionospheric plasma parameters $\omega_{\mathrm{pe}} \approx \omega_{\mathrm{ce}}$ when the Doppler shifted wave velocity

$$
V_{D R}=\frac{\omega-\omega_{c}}{k} \sim \frac{\omega_{p}-\omega_{c}}{\omega_{p}} V_{b}
$$

falls into thermal electron speed and resonates with bulk electrons. In our case $\omega_{\mathrm{pe}}<\omega_{\mathrm{ce}}$ and the velocity (4) is out of the region of plasma distribution function.

We shall investigate another hypothesis that the damping of oblique Langmuir waves may be explained by the influence of transverse limitation of the beam width. The electron beams which generate Langmuir waves appear to be related to Alfvenic structures which have a transverse size of 50-500 m, comparable to the inertial electron length $\lambda_{e} \sim c / \omega_{p}$ at Freja altitudes. Effective damping caused by Langmuir wave emission from the beam region can be estimated as $v_{\text {eff }} \sim V_{g} / R_{b}=A\left(V_{b} / R_{b}\right) \theta$, where $R_{\mathrm{b}}$ is the beam width and the group velocity $V_{\mathrm{g} \perp}$ implied by (3) is $V_{\mathrm{g} \perp} \sim A V_{\mathrm{b}} \theta$. The wave damping limits the sphere of instability by the condition $\gamma \geq v_{\text {eff. }}$. For the parameters indicated in the Table 1 and $R_{\mathrm{b}}=300 \mathrm{~m}$, we find that $\theta_{\max } \sim 0.1 \mathrm{rd}$, and $\Delta \omega \sim 0.01 \omega_{p}$ which corresponds well with the observed width of Langmuir wave spectrum.

\section{PARAMETRIC DECAY $\mathrm{L}_{1} \rightarrow \mathrm{L}_{2}+\mathrm{LH}$; EXPERIMENTAL BACKGROUND}

Short duration of the HF snapshots $(1 \mathrm{~ms})$ and frequency response of the filter in HF channel make it impossible to analyze waves in a range from 1 to $10 \mathrm{kHz}$ on the basis of $\mathrm{HF}$ channel only. Spectrum taken from both HF and MF (sampled at $32 \times 10^{3} \mathrm{~s}^{-1}$ ) clearly shows enhanced wave power near LH frequency $(1-10 \mathrm{kHz})$ together with strong peak at plasma frequency (see Figures 2 and 4 in Stasiewicz et al., 1996). Due to design of the F4 instrument on Freja data cannot be sampled in both channels simultaneously and therefore a time lag exist between two measurements, making it impossible to make a direct comparison of HF and MF waveforms. But it is possible to make a statistical comparison of wave activity around LH frequency and modulation frequency of Langmuir waves, under assumption that Langmuir and LH activity usually observed in localized region related to large-scale Alfvénic turbulence and plasma parameters should not vary much during fraction of second between neighbor HF and MF measurements. We present a comparison of such two measurements in Figures 2. Solid line represent region of enhanced wave activity around LH frequency measured in MF channel and asterisk show the location of modulation frequency observed in HF channel. In almost 2/3 cases modulation frequency is within the region LH activity. The rest of the data may be explained by the assumption that HF and MF measurements are done for different regions due to high spacecraft velocity $(7 \mathrm{~km} / \mathrm{s})$ and fine localization of Langmuir activity. 
Fig. 2. LH frequency range (solid line) and modulation frequency $(*)$ for two neighbor snapshots in MF and HF channels.

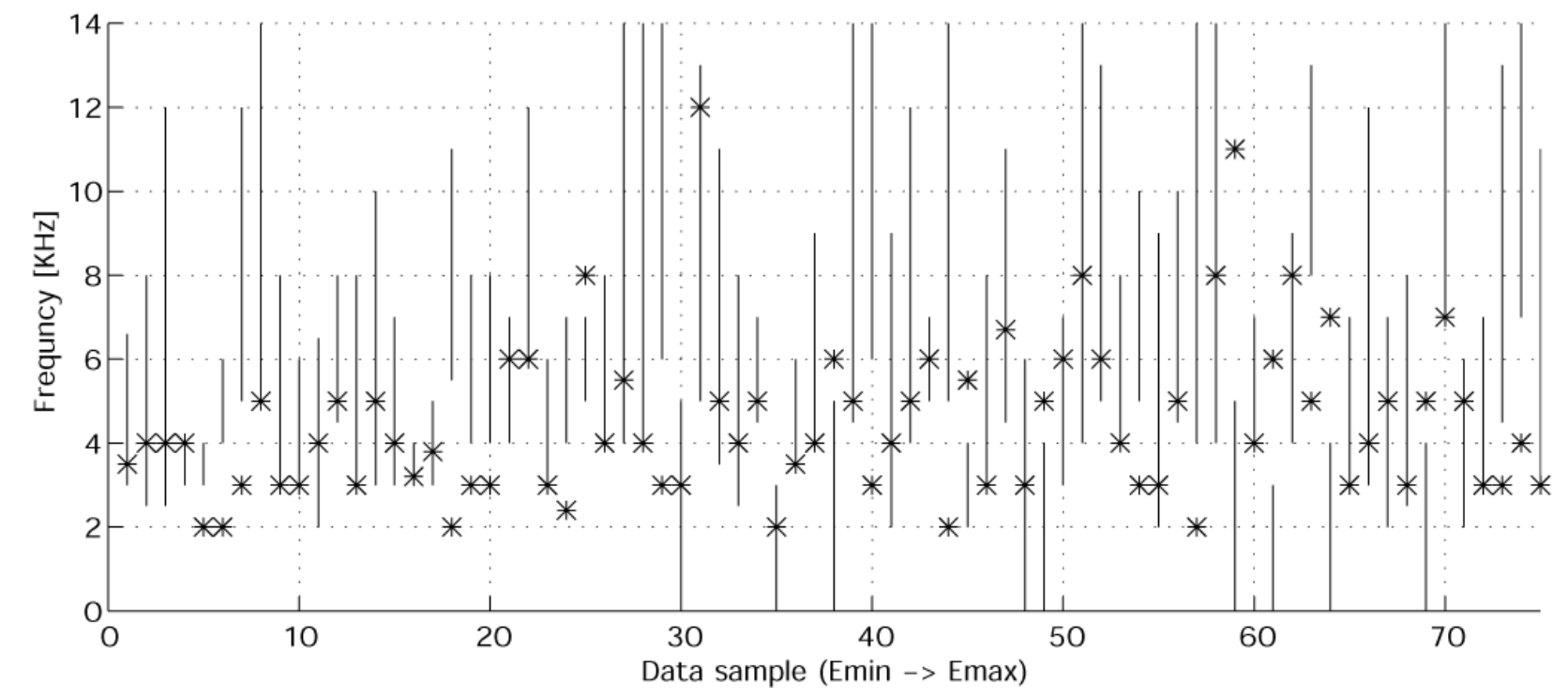

\section{PARAMETRIC DECAY $\mathrm{L}_{1} \rightarrow \mathrm{L}_{2}+\mathrm{LH}$; THEORY}

The equations for the non-linear wave-wave interaction for the Langmuir waves as well as for the lowerhybrid waves have been written in the literature in quite general form (Sturman,1976, Ergun et al.,1991). In this section we present the analysis relevant to the Freja experimental conditions. Let us consider a case where the primary wave is generated by the beam and secondary harmonics appear as a result of parametric decay (or decay cascade) of the primary wave to Langmuir and low-frequency plasma wave. In the case $T_{\mathrm{e}}<T_{\mathrm{i}}$, the ion-acoustic wave is strongly damped and the proper low-frequency mode is lower-hybrid wave as indicated by Freja observations (Stasiewicz et al.,1996, Bonnell et al.,1997). We should mention here that the short duration of the HF snapshots $(1 \mathrm{~ms})$ and fast motion of the satellite through the interaction region does not make it possible to identify ion-acoustic type modulations in the experimental data.

The novelty of further consideration lies in fact that we take into account the transverse limitation of auroral electron beam. We prove that radiation losses caused by the wave emission from the beam region lead to the selection of separated secondary modes in the triplet of parametrically connected waves. The final outlook of equations for three-wave interaction derived after too complicated but standard calculations is:

$$
\begin{aligned}
& \frac{\partial}{\partial t} \varphi_{1}=-i \beta_{1}(\theta) \varphi_{2} \varphi_{3}^{*}, \\
& \frac{\partial}{\partial t} \varphi_{2}=-i \beta_{1}(\theta) \varphi_{1} \varphi_{3}^{*}-v_{2}(\theta) \varphi_{2}, \\
& \frac{\partial}{\partial t} \varphi_{3}=-i \beta_{3}(\theta) \varphi_{1} \varphi_{2}^{*}-v_{3}(\theta) \varphi_{3} .
\end{aligned}
$$

Here $\varphi_{1,2,3}$ are the potentials of interacting waves (connected with the amplitude of electric field by $\vec{E}_{j}=-i \vec{k}_{j} \varphi_{j}, j$ $=1,2,3), \theta$ is the direction of the secondary Langmuir wave propagation toward the magnetic field, $v_{j}$ are the radiation damping rates: $v_{2}=V_{g \perp 2} / R_{b}=A\left(V_{b} / R_{b}\right) \theta, \quad v_{3}=V_{g \perp 3} / R_{b}=\left(g(\theta) / 2(1+\alpha) \cdot v_{2}, \alpha \equiv \omega_{p}^{2} / \omega_{c}^{2}\right.$, anglefunction $g(\theta) \equiv\left[(1+\alpha)\left(1-\theta_{\min }^{4} / \theta^{4}\right)\right]^{1 / 2}$, cut-off angle $\theta_{\min }^{4} \equiv 4(m / M)(1-\alpha)^{2} /(1+\alpha), \beta_{j}$ are the coefficients of three-wave coupling: $\beta_{1}=\left(e / 2 m V_{b}\right) k_{0} \theta g(\theta), \beta_{3}=\left(e / 2 m V_{b}\right) k_{0}(\theta g(\theta)+\alpha) /\left(1-\alpha^{2}\right), m$ is the electron mass and $M$ is the ion one, $k_{0}=\omega_{p} / V_{b}$ is the wave number of primer Langmuir wave. 
Being defined, the equation set (5) may be solved both analytically and numerically. The left panel of Figure 3 displays the behavior of decay instability growth rate $\gamma=\gamma(\theta)$ derived from Eq. 5. The local extremum of the growth rate appears at small $\theta$-angles due to the dissipation of the waves (terms $\propto v_{2,3}$ in Eq. 5).

The right panel of Figure 3 represents the waveform $E(t)=E_{1}(t)+E_{2}(t)$ calculated for the values of plasma parameters indicated in Table 1, the initial amplitude of primary wave $E_{1}(t=0)=200 \mathrm{mV} / \mathrm{m}$, and the optimal value of the $\theta$ - angle (corresponding to the maximal growth rate). On the Figure 3 one may notice three characteristic time scales of the problem: 1) the high frequency filling of wave packets with frequency close to $\left.\omega_{p}, 2\right)$ the beatings with the difference frequency $\omega_{1}-\omega_{2} \sim 0.02 \cdot \omega_{p}$, and 3) the slow modulation of beatings connected with the exchange of energy between interacting Langmuir waves. Let us stress the good coincidence of calculated wave form $E(t)=E_{1}(t)+E_{2}(t)$ with the experimental ones.
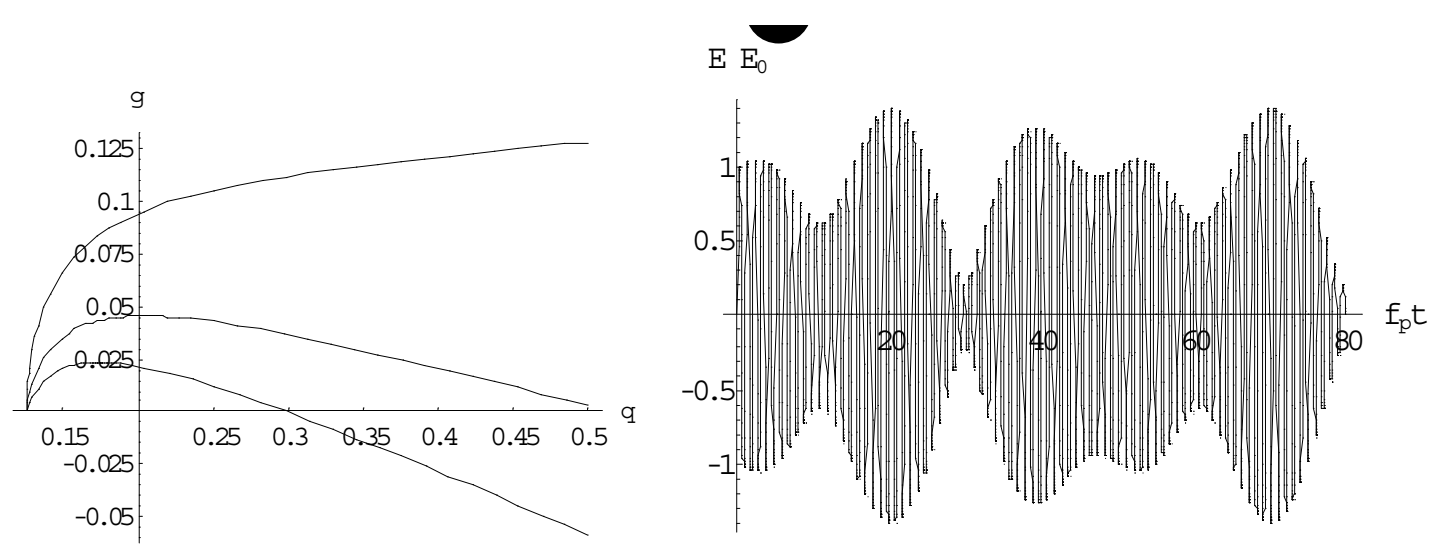

Fig.3. Left panel: decay instability growth rates versus the direction of the secondary Langmuir wave propagation. Right panel: Langmuir waveform $E=E_{1}+E_{2}$ defined by Eq. (5).

\section{CONCLUSION}

The generation of Langmuir wave packets is the common phenomenon registered in laboratory and space conditions with a great diversity of plasma parameters. We have quantitatively investigated one of the most believable mechanisms - the parametric decay of the beam driven Langmuir wave to the secondary one and low frequency wave. Our examination is based of the FREJA observational data and relates to the conditions met in upper polar ionosphere, including the quite peculiar inequalities: $\omega_{p e}<\omega_{c e}, T_{e}<T_{i}$. Under such conditions the decay scheme is to be $\mathrm{L}_{1} \rightarrow \mathrm{L}_{2}+\mathrm{LH}$. The peculiarity of this parametric process is related to the fact that under the condition $\omega_{p e}<\omega_{c e}$ both lower-hybrid and Langmuir modes belong to the same dispersion branch of plasma waves: $\omega_{l h}<\omega<\omega_{p e}$. Langmuir oscillations $\omega=\omega_{p e}$ in this case are not separated from oscillations with lower frequencies which is why three wave decay could take place in a wide range of the difference frequencies $\omega_{3}=\omega_{1}$ $-\omega_{2}$.

We have shown that the transverse limitation of electron beams propagating in the polar ionosphere causes the selection of separated secondary harmonics from the continuous spectra of generated Langmuir and lowerhybrid waves. The waves emission from the beam region causes radiation dissipation with effective damping rate $v_{\text {eff }} \sim \theta$. As result, the primary Langmuir wave decays to the slightly oblique Langmuir wave. Beating of the primary and secondary Langmuir waves produces waveforms displayed on Figure 5. Such types of modulated wave packets have been observed in FREJA experiments.

\section{REFERENCES}

Akimoto, K., Y. Omura, and H. Matsumoto, Rapid generation of Langmuir wave packets during electron beamplasma instabilities, Phys. Plasmas, 3, 2559-2563, 1996.

Bonnell, J., P. Kinter, J.E. Wahlund, and J. Holtet, Modulated Langmuir waves: observations from FREJA and SCIFER, J. Geophys. Res., 101, 17233-17240, 1997. 
Christiansen, P., V. Jain, and J. Bond, Laboratory beam-plasma interaction - linear and nonlinear, in Artificial particle beams in space plasma studies, ed. by Grandal, pp. 439-470, Plenum Press, 1982.

Ergun, R.E., C.W. Carlson, J.P. McFadden, J.H. Clemmons, and M.H. Boehm, Evidence of a transverse Langmuir modulation instability in space plasma, Geophys. Res. Lett., 18, 1177-1181, 1991.

Forme, F., A new interpretation of the origin of enhanced ion acoustic fluctuations in the upper ionosphere, Geophys. Res. Lett., 20, 2347-2350, 1993.

Gurnett, D.A., G.B. Hospodarsky, W.S. Kurth, D.J. Williams, and S.J. Bolton, The fine structure of Langmuir waves produced by a solar electron event, J. Geophys. Res., 98, 5631-5637, 1993.

Kellog, P.J., K. Goetz, R.L. Howard, and S.J. Monson, Evidence for Langmuir wave collapse in the interplanetary plasma, Geophys. Res. Lett., 19, 1303-1306, 1992.

Kojima, H., H. Furuya, H. Usui, and H. Matsumoto, Modulated electron plasma waves observed in the tail lobe: Geotail waveform observations, Geophys. Res. Lett., 24, 3049, 1997.

Muschietti, L., Roth, and R. Ergun, Kinetic localization of beam-driven Langmuir waves, J. Geophys. Res., 100, 17481-17490, 1995.

Newman, D.L., M.V. Goldman, R.E. Ergun, and M.H. Bohem, Langmuir turbulence in the auroral ionosphere: 1, linear theory, J. Geohys, Res., 99, 6367-6376, 1994.

Nezlin, M.V. Observation of Langmuir solitons, in Plasma Physics, Advances in Science and Technology in the USSR, ed. by B. Kadomtsev. pp. 144-175, MIR Publisher, Moscow, 1981.

Stasiewicz, K., B. Holback, V. Krasnoselskikh, M. Boehm, and P. Kinter, Parametric instabilities of Langmuir waves observed by FREJA, J. Geophys. Res., 101, 21515-21525, 1996.

Sturman B.I. Plasma turbulence near the lower-hybrid resonance. Sov. JETF, 71, 613-620, 1976. 